\title{
Las diferencias en el desarrollo moral en función del sexo: Un estudio realizado en el País Vasco
}

\section{Concepción Medrano}

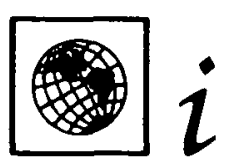

La autora de este artículo, presenta datos empiricos que refutan la crítica de Gilligan al instrumento de medida utilizado por Koblberg. A través de la investigación que presenta, no se puede confirmar la existencia de diferencias entre mujeres y bombres en cuanto al razonamiento moral. Los valores recogidos en los dilemas reales que los sujetos nos relatan, representan un material muy valioso, para planificar y desarrollar los contenidos transversales relacionados tanto con la coeducación, como con la educación moral.

Las diferencias en el desarrollo del juicio o conductas morales en función del sexo, se ha estudiado desde distintas perspectivas. De alguna manera, se podría decir que la mayoría de las investigaciones arrojan diferencias entre mujeres y hombres presentando una superioridad estos últimos sobre las mujeres.

Si consideramos que, en la sociedad occidental los ambientes y los contextos en los que hombres y mujeres desarrollamos nuestras capacidades, aún en la actualidad suelen ser diferentes, estas diferencias pueden en muchos casos, atribuirse al ambiente o modelos de internalización, con los que se ha interactuado, más que al hecho de pertenecer a uno u otro sexo.

Existen múltiples investigaciones, realizadas desde modelos y perspectivas distintas e incluso desde ideologías diferentes que nos conducen a realizar una reflexión acerca de este tema tan polémico como interesante, Bull (1976) respecto a esta cuestión ha señalado.

«Los estudios sobre el criterio moral no revelaron la existencia de una clara distinción entre los sexos. Ofrecieron indicios de que las chicas desarrollan la conciencia antes que los chicos, pero se trata de la tendencia que siguen, más que de pruebas definitivas» (pág. 113).

En una primera revisión acerca de estas diferencias, se pueden establecer distintas perspectivas para analizar los datos de las distintas investigaciones realizadas: el enfoque psicoanalítico, el enfoque del aprendizaje social y el enfoque cognitivoevolutivo. 
Nuestro interés en este trabajo, es analizar este área psicoeducativa desde el modelo estructuralista, que centra su atención en la actividad estructuradora del sujeto, pero considerando también la importancia del medio ambiente. El enfoque cognitivo-evolutivo es en concreto, el marco teórico en el que se presenta el análisis de los datos que se han recogido. Antes de presentar el mismo, señalemos brevemente algunas ideas defendidas desde otras pespectivas y datos de algunas de las investigaciones, que apoyan las diferencias de sexo en el desarrollo moral.

El modelo psicoanalítico, atribuye a la mujer un superyo, una conciencia moral más débil que la del hombre. Desde la perspectiva freudiana, en la mujer, como consecuencia de la diferencia anatómica de los sexos, el complejo de Edipo toma un carácter diferente al masculino. El temor a la pérdida del amor de la madre como factor desencadenante, no posee la fuerza de la angustia de castración en el varón, por lo que en la niña la conciencia moral, no alcanzará la fuerza del varón. (Etxeberría 1989.)

Algunos autores dentro de este modelo, han reflexionado acerca de la distinta posición social de cada sexo y la importancia que ello conlleva en los procesos de identificación por parte de la mujer. La manera de comportarse por parte del progenitor más fuerte es mejor imitada, tanto por niños, como por las niñas. El tipo de prácticas educativas utilizadas con cada sexo, apuntaría hacia una explicación más rigurosa de estas diferencias, más allá del determinismo biológico y cultural que presenta este enfoque.

En este sentido, es necesario considerar siempre la dimensión socio-cultural en el aprendizaje de nuestro rol sexual y reflexionar acerca de los cambios sociales que en la actualidad se están produciendo. Estos cambios pueden alterar los procesos de identificación de las mujeres. Pensemos por ejemplo: en los modelos de identificación que pueden desarrollarse cuando el progenitor masculino asume el trabajo de la casa y cuida a sus hijas/os, mientras que sus mujeres trabajan fuera para mantener económicamente a la familia.

Naisbit (1.983) al realizar un análisis de macrotendencias concluye que el rol de la mujer, no será en el futuro el mismo que ha venido desempeñando históricamente, lo cual no deja de crear inquietud en ciertos sectores sociales.

En cuanto al enfoque del aprendizaje social, que ha subrayado también la importancia de los modelos materno y paterno como refuerzos para el aprendizaje y desarrollo de las capacidades humanas, hay que destacar la importancia que ha concedido a las estructuras medio-ambientales. Las experiencias vicario-simbólicas aportan una explicación no organicista de las diferencias, poniendo el acento en el ambiente como estimulador y conformador de los aprendizajes tanto convencionales como morales. En las investigaciones llevadas a cabo dentro de este enfoque (Bandura y MacDonald 1.963) las mujeres presentan más dependencia del juicio de los demás y de la autoridad que los varones.

En cierto sentido, se podría interpretar por los datos precedentes de los modelos psicoanalítico y del aprendizaje social, que las mujeres presentamos una mayor debilidad moral y dependencia de los demás en comparación con el otro sexo.

Una de las limitaciones más criticadas a estos últimos enfoques, es el reduccionismo que conlleva, la explicación del proceso de desarrollo a la imitación de los aspectos externos, medibles y observables, sin tener en cuenta los procesos intermedios en la construcción de los valores morales. Estos enfoques han resultado insuficientes para poder comprender la formación de valores autónomos, dónde en muchos contextos y situaciones, es necesario transgredir las normas aceptadas por el grupo de pertenencia.

$\mathrm{Si}$ pasamos a analizar los datos que poseemos en los modelos estructuralistas, dentro del enfoque congitivo-evolutivo, Piaget (1.932) destacó también el hecho de que las niñas codifican las reglas del juego más tarde y manifiestan menos interés que los niños. 
Sin embargo, en este tipo de valoraciones es muy importante acotar el contenido de la propia situación. ¿Son las reglas del juego situaciones-estímulos morales? o ¿se están analizando contenidos que son más interesantes para los chicos que para las chicas?

Antes de establecer este tipo de conclusiones es necesario cambiar los contenidos de la misma prueba, introduciendo juegos que resulten motivadores tanto para las niñas como para los niños. Magowan y Lee (1.970) interpretan como un error instrumental las conclusiones de Piaget y señalan la necesidad de variar no sólo el sexo del protagonista de la historia, sino el número de protagonistas de uno y otro sexo.

Freeman y Giebinik (1.979) encuentran un razonamiento moral más avanzado en los chicos, cuando el protagonista es del mismo sexo. En la misma línea, Bussey y Maughan (1.982) en sus investigaciones con varones, afirman que el razonamiento es superior si el protagonista es masculino.

En un análisis global de las conclusiones de estos trabajos, se puede deducir que el instrumento de medida en muchas ocasiones se convierte en un sesgo importante a la hora de establecer las diferencias.

\section{LA PERSPECTIVA DE KOHLBERG E INVESTIGACIONES REALIZADAS A PARTIR DE SU TRABAJO}

El próposito de Kohlberg no consistió en establecer diferencias entre hombres y mujeres, sin embargo, de su trabajo se han derivado muchas conclusiones como la de asociar al estadio tercero las respuestas de las mujeres, al presentar una orientación hacia la empatía y preocupación por los demás. El mismo Hoffman (1.977), gran conocedor de este tema, afirma en otro contexto diferente al que estamos recogiendo en este artículo, que las mujeres tienen una tendencia general a ponerse en el lugar de otro, es decir a la empatía, lo cual no debe de significar un estadio más bajo, estructuralmente hablando.

No vamos a explicar los estadios de razonamiento moral de Kohlberg, ya que en este momento poseemos muchos trabajos en castellano dónde el lector puede encontrar una explicación detallada y exhaustiva (Diaz-Aguado 1.982, 1.989; Medrano 1.986, 1.990; Puig y Martinez 1.989; Perez-Delgado y García- Ros 1.991; Martinez y Puig 1.991; Kohlberg 1.992; Diaz-Aguado y Medrano 1.994) de la perspectiva cognitivo-evolutiva en el caso del razonamiento moral.

Destacar sólamente que esta perspectiva se caracteriza a diferencia de otros enfoques, por estudiar las transformaciones estructurales que se producen a lo largo del desarrollo humano; defendiendo que son construcciones que el sujeto realiza en interacción con las estructuras ambientales y que siguen una secuencia evolutiva universal.

Esta secuencia evolutiva se manifiesta en tres niveles de desarrollo, según la relación que el sujeto estableza con las expectativas y reglas del grupo/sociedad (convención). El nivel preconvencional, dónde las reglas sociales son externas al yo; el nivel convencional, cuando se produce una identificación con las expectativas de los demás; y el nivel postconvencional, que se alcanza cuando el individuo defiende los valores construidos por el mismo y que interpreta como básicos para el desarrollo de una sociedad justa. Cada uno de estos tres niveles, comprende dos estadios, que explican los seis estadios de razonamiento moral de Kohlberg.

Si se tiene.en cuenta los datos empíricos de las distintas investigaciones que se han realizado, en la línea del modelo de Kohlberg, se puede afirmar:

1. Que no existen diferencias estructurales en función del sexo, pero si aparecen diferencias en la orientación del contenido.

2. Las diferencias encontradas en adultos, con una superioridad de los hombres sobre las mujeres, se explica más por el nivel de educación y ocupación que por las diferencias de sexo. 
La mayoría de estas investigaciones concluyen acerca de la necesidad de profundizar en otros factores como experiencias socio-morales o prerrequisitos cognitivos. En sentido Walker señala (1.984):

«La revisión de la literatura, resulta clara respecto a la similtud entre el razonamiento moral de hombres y mujeres... Quizá haya llegado el momento de centrar nuestra atención en otros aspectos como el papel de los prerrequisitos cognitivos, las experiencias socio-culturales que facilitan el desarrollo y en el estudio de la relación entre el razonamiento moral con las emociones y la conducta» (pág. 688)

Sin embargo, desde finales de los años sesenta y a partir del trabajo de Kohlberg (1.958), una mujer, Carol Gilligan alumna y colega del primero en la Universidad de Harvard, presenta una dura crítica al instrumento de medida utilizado por Kohlberg y sus colaboradores en las distintas investigaciones longuitudinales y transversales.

Gilligan (1.982) considera que algunas características del pensamiento femenino sobre la responsabilidad, el bienestar, el cuidado de los demás etc.. se infravaloran en el sistema de calificación de Kohlberg, dado que se interpretan como estadios más bajos los valores que desde el punto de vista tradicional se han atribuido al hombre. Estos valores se refieren a: la abstración, la racionalidad, el pensamiento hipotético deductivo, etc.. los cuales se han calificado como estadios más altos. Esta autora ha propuesto una investigación alternativa para poder conocer la secuencia invariante de desarrollo moral en la mujer.

Aunque no disponemos de datos empíricos, que puedan apoyar la hipótesis de Gilligan y comprobar las críticas realizadas al instrumento de Kohlberg, ella ha realizado una observación sobre la progresión de estadios morales en la mujer en una investigación sobre el aborto. Al referirse a las orientaciones que las mujeres ofrecen para sus razonamientos morales, explica:

«El problema moral para las mujeres, surge de un conflicto de responsabilidades más que de una competencia de derechos y ésto tiene que ver con la comprensión hacia los demás y con la manera de entender la responsabilidad y las relaciones con los demás». (1.982, pág.19)

La investigación de Gilligan acerca del aborto, nos parece uno de los aspectos más importantes a destacar, en cuanto a la reclamación de "contenidos» propios de la mujer, a la hora de establecer una valoración sobre el razonamiento moral.

Más recientemente y en nuestro contexto Moltó Brotons; Perez-Delgado; Mestre-Escrivá y Garcia Ros (1.990) han investigado las diferencias de razonamiento en mujeres y hombres a través del DIT (Rest 1.979) en una muestra formada por 1.174 sujetos. Estos autores no encuentran diferencias significativas y concluyen que no existe base científica para sostener que las mujeres razonan por debajo de los hombres. Más bien, defienden que según sus datos existe una marcada tendencia a favor de las mujeres, ya que encuentran una mayor presencia de razonamiento postconvencional en éstas, una vez controlado el efecto de la edad.

Las conclusiones a las que llegan este grupo de investigadores de la Universidad de Valencia, coinciden con los resultados obtenidos a través de DIT en anteriores trabajos. (Rest 1.979; Perez-Delgado, Mestre y García-Ros 1.990).

\section{Los dilemas reales: estructura y contenido en la secuencia evolutiva de razonamiento moral}

Continuando en la línea de pensamiento kohlberiano, nos ha parecido adecuado conocer el tipo de valores que en función del sexo aparece en la secuencia evoluti- 
va desde los 8 hasta los 25 años, en los dilemas reales que los sujetos relatan; y analizar si existen diferencias a nivel estructural (estadio de razonamiento moral).

En el sistema de calificación de Kohlberg, la estructura se refiere al razonamiento o argumentación del sujeto que nos lleva a situarle en un estadio de juicio moral, mientras que el contenido se refiere a los valores en conflicto que aparacen en cada dilema. Por ejemplo: Jon de 14 años nos relata la siguiente situación:

«Mi problema es que mis amigos a veces se burlan de una profesora nueva que ha venido y le hacen bastantes trastadas, yo creo que no está bien lo que hacemos porque yo también me meto en el lío, pero cuando les digo que yo no quiero continuar haciendo lo que hacemos, es como si me rechazaran y me dejan a un lado, entonces el problema es qué hacer, yo necesito que mis amigos me acepten y también quiero hacer cosas para llevarnos bien, pero me parece mal no respetar a la nueva»

Jon presenta un nivel de razonamiento moral convencional, concretamente sus argumentos presentan las características del estadio tercero, y los valores o contenido del conflicto se centra en: la fidelidad a los amigos y el respeto a la nueva profesora.

Una de las críticas realizadas al modelo cognitivo-evolutivo de Kohlberg (Medrano 1.986), se ha basado en la poca importancia concedida al contenido o a las propias situaciones contextuales para explicar el desarrollo moral. Por esta razón, es necesario trabajar no sólo con dilemas hipotéticos sino con los dilemas estructurados y construidos por el propio sujeto.

Considerando esta crítica, se han recogido los dilemas reales de sujetos con edades comprendidas entre 8 y 25 años pertenecientes a la Comunidad Autónoma Vasca. El interés de este trabajo se dirige al tipo de dilemas que estructuran los sujetos, por varios motivos:

1. Las implicaciones psicopedagógicas para el desarrollo de las estrategias de enseñanza-aprendizaje; fundamentalmente para trabajar contenidos transversales, referidos a la educación moral. 2. La validez ecológica de los datos recogidos. 3 . El análisis que se puede realizar para investigar las relaciones entre el contenido del dilema y la estructura de razonamiento. 4 . El conocimiento de los dilemas que surgen en nuestro contexto cultural y las posibles comparaciones con otros contextos diferentes. Y por último, y ésta es la razón del estudio que aquí presentamos: el análisis que permite realizar de las diferencias en función del sexo.

\section{Procedimientos y resultados}

Para presentar los datos de los que disponemos, hemos agrupado las edades cada tres años, de forma que resultan los cinco grupos siguientes: 1) de 8 y 9 años; 2) de 12 y 13 años; 3) de 16 y 17 años 4) de 20 y 21 años y 5) de 24 y 25 años. En cada grupo se ha trabajado con una muestra de 25 hembras y 25 varones, resultando en total 250 sujetos. Se ha comenzado con la edad de 8 años, debido a que el propio método de Kohlberg, no se puede utilizar con sujetos anteriores a esa edad. Tanto la estructura del dilema hipotético, como la descripción de un dilema real es difícil de procesar y estructurar antes de los 8 o 9 años.

La recogida de datos se ha realizado a través de una entrevista clínica individual, de una duración aproximada de media hora, en la que se comenzaba con un dilema hipotético, para posteriormente pedir al sujeto que nos estructurara un dilema real. El dilema hipotético seleccionado para este trabajo fué el dilema $\mathrm{V}$ de la Forma B ( Diaz-Aguado y Medrano 1.994). En este dilema los valores que aparecen en conflicto son la vida y la autoridad (respetar la autoridad de un capitán en situa- 
ción de guerra o la pérdida de una vida frente a la vida de los hombres de toda una compañia de marines).

Al comenzar con un dilema hipotético, nos asegurábamos de que el sujeto había comprendido lo que era un dilema moral, es decir, un conflicto de valores; para posteriormente a través de la entrevista, profundizar en los argumentos que explicaran las elecciones de sus respuestas. De esta forma, es más fácil pedir al sujeto que nos relate una situación parecida a la anterior (la hipotética), dónde ellos o ellas no tuvieran claro que era lo que se debía de hacer e interrogarles acerca de cómo se sabe lo que uno debe de hacer, en situaciones de conflicto real.

El mismo dilema hipotético se pasó a todas las edades, aunque con los sujetos más pequeños, fué preciso asegurarnos bien de que lo habian entendido; por lo que ha sido necesario repetirlo más de una vez.

La corrección basada en el manual de Colby y Kohlberg (1.987), se ha realizado por dos jueces con el fin de poder asegurar la fiabilidad en la asignación de los estadios morales. En este trabajo, únicamente se presentan los datos del dilema real expuesto por cada sujeto. No se presenta ningún análisis respecto al nivel de razonamiento en el dilema hipotético, dado que nuestro interés se ha centrado en observar si existen diferencias en función del sexo en el dilema estructurado por ellos mismos.

I. Respecto al primer grupo, los sujetos de 8 y 9 años, no presentan diferencias en cuanto a la temática planteada por ambos sexos. Aproximadamente la mitad de los niños (15) y niñas (12) plantean «las relaciones de autoridad» bien sea con sus padres, educadores etc..como dilema real. Por ejemplo María (9años) nos relata:

«Un día mi madre me pidió que cuidara de mi hermano pequeño, pero había unos dibujos animados en la T.V. y no le hice mucho caso, luego se puso a llorar y me castigaron a mí por su culpa" ¿y tú cómo sabes en situaciones como ésta, qué es lo que bay que bacer?" "pues pienso que la culpa fue de mi hermano, pero hay que hacer lo que mande mi madre, porque ella se preocupa de mí y nos cuida, yo tengo que obedecerla"

El resto de los sujetos, sin diferencias de sexo plantea problemas relacionados con los iguales,(10 niñas y 8 niños) y con el robo (2 niños y 3 niños) Por ejemplo, Javier de 8 años nos describe el siguiente dilema:

«En el comedor se forman dos grupos de amigos, unos se ponen juntos y el otro grupo en otra mesa. Yo con unos juego en el patio y me llevo bien y con los otros, que viven en mi urbanización también. Entonces no sé lo que hacer, si me voy con unos se enfadan los otros y es un lio para mí" ¿quécrees que debes bacer en situaciones como éstas?" "Ir unas veces con unos y otras con otros para que no se enfanden y llevarme bien con todos".

Si nos fijamos en el razonamiento estructural, es decir, el estadio moral utilizado en sus argumentos encontramos: que el $80 \%$ de los niños y niñas se encuentran en el nivel preconvencional (estadio 1, 12 niñas y 15 niños; estadio 2, 9 niñas y 4 niños); el restó de la muestra alcanza niveles transitorios del estadio dos al estadio tres, sin presentar aún niveles convencionales.

Tampoco se pueden establecer diferencias en cuanto a la estructura de razonamiento en función del sexo; desde un análisis más cualitativo, se observa una pequeña diferencia a favor de las niñas, ya que son más las que nos dan argumentos del estadio segundo.

II. En cuanto al segundo grupo, con edades comprendidas entre 12 y 13 años, tampoco hallamos diferencias entre los sexos, en cuanto a los contenidos planteados, ni en el razonamiento moral. Respecto a éste se sitúan en niveles convencionales el $80 \%$ de la muestra. En el estadio tres ( 17 chicas y 18 chicos ) y en el estadio 4 (el $10 \%$ de los sujetos). En el estadio 2 del nivel preconvencional se sitúa el $20 \%$ de los sujetos restantes. En términos globales no existen diferencias significativas entre uno 
y otro sexo; solamente destacar que el estadio cuarto es alcanzado por tres chicos de 14 años y una chica de la misma edad.

Si analizamos el contenido de los dilemas, el 60\% (16 chicas y 18 chicos) de la muestra relatan situaciones dónde aparece un conflicto entre la autoridad de los padres, profesores, monitores, incluso hermanos mayores y su propia opinión o su sentido de lo correcto. Por ejemplo Cristina (13 años) relata:

«Estando en clase, vino el profesor y me castigó por algo que no había hecho. El problema fué que era su palabra frente a la mía, o mejor dicho, frente a toda la clase» ¿por qué esta situación es un conflicto? "Porque toda la clase decía que yo no había hecho nada y somos cuarenta y por otro lado, él es el profesor y decía que tenía que ir fuera de clase cuando no tenía razón" ¿qué crees que se debería bacer en esa situación? "Yo creo que nuestra opinión también deberá contar, somo personas ¿no?..tiene que valer lo que diga la clase, además luego se trabaja mejor" $¿ Y$ la opinión del profesor? "También es importante, a veces demasiado importante, pero él tiene que dar ejemplo y si nos dicen que es importante respetarse pues que respeten ellos. En este caso, no se tuvo en cuenta la opinión nuestra y yo creo que somos personas como los demás, tenía que escuchar nuestras razones». ¿ ¿ Debe contar igual vuestra opinión y la del profesor?" "Depende, por ejemplo, si es para hacer el vago y él dice que hay que trabajar, no, pero en otras cosas que pasan en clase y no están relacionadas con el estudio, son más cosas de personas, entonces nuestra opinión es importante. Un profesor es un hombre y se puede equivocar. Tiene que oir a los alumnos, cuando todos dicen lo contrario a él».

Este tipo de contenido, como el relatado por Cristina se repite en muchos sujetos, tanto chicos como chicas, en relación a padres y cualquier figura de autoridad, aunque el más frecuente es las relaciones profesores-alumnos.

El resto de los sujetos decribe situaciones relacionadas con:el robo ( 3 chicos y 3 chicas); el dilema del nacionalismo-terrorismo (5 chicos y 4 chicas) y el aborto ( 2 chicas). Por ejemplo Kepa (14 años):

«A veces, no me parece bien que se vayan poniendo bombas por ahí, para defender a Euskadi. Mira lo que pasa, a veces, que pueden morir niños. Si los de Madrid no nos quieren dar nuestra autonomía y dejarnos lo nuestro, hay que luchar contra ellos porque no les gusta que hablemos nuestra lengua. Todos los políticos no hacen nada más que hablar y chulear, por eso algunas veces está bien un poco de lucha armada, pero creo que se están pasando" ¿Cuál es el dilema?" «Por un lado, el Parlamento es un rollo y lento, hay que hacer algo, pero por otro no se puede matar a niños" ¿Cómo sabes lo que se debe de hacer en conflictos de este tipo? "Yo creo que no se puede pedir la libertad de Euskadi, matando gente, no sé, es pedir la libertad, pero tú la quitas, si no hay vida, pues no hay nada"

La temática de nacionalismo-terrorismo, dado que todos los sujetos entrevistados pertenecen al contexto del Pais Vasco, se repite en todas las edades, aunque a medida que aumenta la edad utilizan más esquemas y argumentos de estadios más altos. En este grupo (13-14 años) ha sido planteado por una minoría de sujetos, sin embargo, lo han relatado tanto chicas como chicos, mientras que en edades superiores se observa una tendencia a ser descrito más por hombres, que por mujeres. También, me parece importante señalar que la temática del aborto, no ha sido expuesta por ningún chico, siendo este tópico el único diferente en función del sexo.Como se podrá observar, esta tendencia se va a generalizar en los otros grupos, hasta los 25 años, siendo uno de los contenidos más específicos y diferenciales entre las mujeres y los hombres. Esta diferencia de contenido, no supone diferencia en la estructura de razonamiento, sino que más bien nos confirma algunas hipótesis respecto a las diferencias en cuanto a intereses, preocupaciones, etc.. que nos pueden ser muy útiles para orientar los debates en el aula, favorecer la interacción entre ambos sexos, planificar las estrategias de intervención en programas de coeducación y en definitiva contextualizar la enseñanza y el aprendizaje a partir de dilemas reales, estructurados por las alumnas y alumnos. 
III. El tercer grupo (16 y 17 años), presenta diferencias en cuanto a la temática de los dilemas reales, que no hemos observado en edades anteriores. El hecho de que sea en este grupo, de 16 y 17 años, donde comienzan a aparecer diferencias significativas en relación al contenido del dilema real, nos aporta una información muy relevante para continuar profundizando, en las implicaciones psicoeducativas desde la propia psicología evolutiva.

No ocurre lo mismo en el nivel de razonamiento, dónde no se hallan diferencias en cuanto a los sexos. El $50 \%$ de la muestra (23 mujeres y 27 hombres) presentan argumentos del estadio tres; el $40 \%$ se sitúa en el estadio cuarto y un $10 \%$ en el estadio quinto; en estos dos últimos estadios se recogen el mismo número de mujeres y hombres.

Respecto a los valores expuestos encontramos que: el $40 \%$ de las chicas nos exponen conflictos relacionados con las relaciones sexuales; el $30 \%$ ( 8 sujetos) con las relaciones personales; 5 sujetos plantean conflictos de droga y las dos chicas restantes conflictos de realciones de amistad.

En el grupo de chicos, encontramos que el $60 \%$ nos presenta conflictos relacionados con la desobediencia civil; del $40 \%$ restante ( 8 sujetos) plantean problemas relacionados con la lealtad en la amistad y los otros 2 sujetos con las relaciones sexuales.

Así por ejemplo Juan de 17 años nos expone:

"Yo estoy en contra de que tengamos que ir a la mili. Me parece una pérdida de tiempo trabajar para algo que estoy en contra. Prefiero hacer otro servicio civil” ¿Qué crees que hay que bacer en situaciones como esta? "Creo que debemos juntarnos y hacer asociaciones para poder protestar y luchar de una maner lógica contra unas leyes que no están bien hechas. No creo que se consigue nada con ir a manifestaciones y gritar, me parece mejor intentar cambiar si se puede las normas o leyes que no nos parezcan justas».

IV. El cuarto grupo (20 y 21 años) continúa presentando diferencias entre mujeres y hombres respecto al contenido de dilemas que nos plantean; sin embargo, esta diferencia no aparece en la estructura de razonamiento. Respecto a éste último el $60 \%$ ( 15 mujeres y 20 hombres) de los sujetos entrevistados se sitúan en un estadio cuarto. Un $10 \%$ ( 3 mujeres y 2 hombres) lo hace en un estadio quinto y el $30 \%$ (8 mujeres y 7 hombres) restante ofrece argumentos del estadio tres.

Si nos fijamos en las temáticas relatadas encontramos que el $50 \%$ de las mujeres (12) plantea conflictos en el ámbito de las relaciones personales por ejemplo: la fidelidad en la pareja, la sinceridad en las relaciones de amistad, la tolerancia en el ámbito familiar etc.. Así Paloma de 20 años nos decribe la siguiente situación:

«Estoy saliendo con un chico desde hace cinco meses, pero a mis padres no les gusta, porque no es de mi ambiente. En realidad pertenecemos a medios distintos, pero a mi me parece que es mi propia vida y tengo derecho a equivocarme" ¿Cuáles son. los.valores entre.los que tienes que elegir? "Por un lado, debo respeto a mis padres, no ciegamente, pues su experiencia en otras situaciones me ha servido, y tampoco quiero que sufran innecesariamente; $y$ luego, está mi libertad y mis decisiones personales. Ellos suelen hablar con nosotros, y nos piden opinión. No sé, es como querer que en mi familia no se desequilibre el ambiente por mi culpa, pero también hacer mi vida, y por eso lo vivo de manera conflictiva».

El $50 \%$ restante, del grupo de las mujeres, nos describe situaciones de aborto ( 9 sujetos ) y de injustica social ( 4 sujetos).

Las temáticas de los hombres se distribuyen de la manera siguiente: el 70\% ( 16 sujetos) plantean conflictos relacionados con el nacionalismo y las estrategias para alcanzarlo. El 30\% restante se distribuye entre las relaciones con iguales (4 sujetos); la injusticia social (3) y las relaciones sexuales (2). 


\section{5}

Como se puede observar, los valores elegidos difieren entre hombres y mujeres, aunque ambos presentan niveles parecidos en cuanto a la estructura de razonamiento. ¿No puede significar este hecho, que en el comienzo de la edad adulta, nuestros valores son diferentes y precisamente en esta diferencia, se encuentra la clave del respeto a la individualidad y singularidad de cada persona?

V. Finalmente en el último grupo de 24 y 25 años, no aparecen diferencias ni en cuanto al contenido, ni en cuanto a la estructura.

Presentan un estadio tercero el $20 \%$ y un estadio cuarto el $60 \%$ de mujeres y hombres, sin diferencias entre ellos. El $20 \%$ restante ( 6 mujeres y 4 hombres) alcanzan el estadio quinto.

Las temáticas que aparecen se pueden agrupar en torno a: tráfico de influencias-puesto de trabajo (13 mujeres y 15 hombres); castigo-narcotraficantes ( 5 mujeres y 3 hombres); desobediencia civil-insumisión ( 1 mujer y 2 hombres). Por ejemplo Ana de 25 años nos expone:

«El dilema que yo tengo es el que tenemos muchos amigos en este momento. Cuando vamos a pedir un trabajo, lo queremos hacer limpiamente y superando o no las pruebas necesarias. Luego te das cuenta que algunas personas con menos méritos que tú consiguen el trabajo ¿por qué? porque han hablado con gente, enchufes erc.. ¿Cuáles son los valores en conflicto? La duda que a mí se me plantea es que estoy en contra de pedir favores, en algunas ocasiones yo también o mis padres lo podiamos haber hecho. Pero por principios pienso que no debe hacerse, y sin embargo, si soy coherente con mis principios, aquí estoy, sin encontrar trabajo. Dudo de que es lo que se debe de hacer, porque hay que comer ilo entiendes?».

\section{Conclusiones e implicaciones para la educación moral}

Los datos anteriores no confirman la existencia de diferencias entre hombres y mujeres, tal y como ha sido defendido por Gilligan. A lo largo de las distintas edades desde los 8 hasta los 25 años, observamos una progresión de estadios morales que aumenta con la edad y que confirma la hipótesis de Kohlberg respecto a la secuencia invariante de estadios.

Donde si aparecen diferencias es en el tipo de dilemas (contenido) que los propios sujetos estructuran. Y esto ocurre en dos grupos de edad: 16- 17 y 20 -21 años.

Las diferencias en los valores elegidos en la adolescencia y comienzo de la edad adulta, nos ofrecen datos importantes para desarrollar proyectos educativos desde la diversidad. Aunque sería conveniente reflexionar desde la psicología evolutiva, el por qué se manifiestan estas diferencias en estos periodos.

En todo caso, estas diferencias resultan enriquecedoras para cualquier proyecto de educación moral; superada ya la vieja discusión de si estas diferencias son biológicas o culturales, estoy de acuerdo con el pensamiento de Camps (1990) cuando escribe:

«aunque no haya funciones específicas, ha habido funciones atribuidas, las cuales han desarrollado disposiciones y actitudes concomitantes. Por ello y sólo por ello pienso que existe un bajage femenino redimible y no despreciable; como algo bueno y valioso para todos.. bueno y valioso para la liberación y el progreso de la humanidad» (pág 132)

En las conclusiones de este trabajo, hay que destacar que los conflictos reales planteados por ambos sexos, se caracterizan más por su convergencia que por su divergencia; y en las edades que aparecen diferencias, observamos una magnífica oportunidad para trabajar desde la complementariedad.

En síntesis, se observa que: 
1. Los problemas de relación con la autoridad (padres, profesores, monitores, hermanos mayores) y con los compañeros de la misma edad, ocupan la mayor parte de la temática de los sujetos más jóvenes (9-10 y 13 -14 años).

2. El tema del nacionalismo-terrorismo preocupa a un gran número de sujetos (a partir de los 16 años) junto con el tráfico de influencias y el castigo para los narcotraficantes. Estos contenidos sustituyen a los de etapas evolutivas anteriores y suponen una mayor interacción con su contexto. También reflejan el momento histórico socio-cultural.

3. En edades anteriores a la adolescencia y en la edad adulta ambos sexos plantean temáticas parecidas, bien sean de tipo personal o social.

4. Los dilemas reales, a medida que aumenta la edad, se estructuran a partir de contenidos que reflejan el contexto socio-histórico.

Los valores recogidos en los dilemas reales de las distintas edades, representan un material muy valioso, para planificar y desarrollar los contenidos transversales, tanto en aspectos relacionados con la educación moral, como en programas de coeducación. El hecho de trabajar con los dilemas estructurados por los propios alumnos y alumnas, contribuye a proporcionar un mayor grado de significación a los procesos de enseñanza-aprendizaje.De los datos de este estudio, no se puede concluir que existan diferencias en el desarrollo moral en función del sexo. Por esta razón, la educación para ambos se debe plantear en lo que se denomina el currículo de las tres $\mathrm{C}$ : cuidado, preocupación y relación (caring, concern, connection).

En este área tan debatida de las diferencias entre sexos, y desde el punto de vista educativo tal y como señala Martinez (1.991) «la igualdad» de las mujeres, no se debería plantear a través de un proceso de identificación con el modelo masculino tratando de conseguir una igualdad en la conducta, intereses y habilidades con respecto al hombre. Antes es necesario construir la propia identidad femenina, o más bien, construir una nueva identidad de sujeto/persona que permita desarrollar características y valores femeninos y masculinos con independencia del sexo; y superar la actual tipificación sexual en nuestra sociedad. De este modo, se podrá lograr una educación que contribuya al desarrollo global de las personas, más allá de los estereotipos de «género", generados en las prácticas de socialización.

\section{Referencias}

BANDura, A., y MC DONALD, F. (1963). The Influence of Social Reinforcement and the Behaviour of Models in Children's Moral Judjement.Journal of Abnormal and Social Psycbology, 67, 274-281.

Bussey, K., y MANGHAN, B. (1982). Diference in Moral Reasoning. Journal of Personality and Social Psychology, 42, 701-706.

BuLI, N. (1976). La educación moral: Estella (Navarra): Verbo-Divino.

CAMPS, V. (1990). Virtudes públicas. Madrid: Espasa Calpe.

COLBY, A., y KoHLBERG, L. (1987). The measurement of Moral Judgement, I-II. Nueva York: Cambridge, University Press.

Díaz-AGUADO, M. J. (1982). La relación entre el tipo de educación escolar y el nivel de razonamiento moral. Tesis Doctoral, Universidad Complutense, Madrid.

Díaz-AGUADO, M. J. (1989). El desarrollo del razonamiento moral: significado y evaluación. En Dir. Etxeberría, Perspectivas acerca del cambio moral, posibles intervenciones educativas. San Sebastian: Servicio Editorial de la Universidad del País Vasco.

DÍAZ-AGUADO, M. J., y MEDRANO, C. (1994). Educación y razonamiento moral:Una aproximación constructivista para trabajar los contenidos transversales. Bilbao: Mensajero.

Diseño Curricular (1992). Comunidad Autónoma Vasca. Departamento de Educación Universidades e Investigación, Vitoria.

Elexpuru, I., y MEDRANo, C. (1994). El desarrollo de los valores desde la perspectiva de la interacción de los modelos de Kohlberg y Hall-Tonna. En Clemente y otros. Intervención Educativa y Desarrollo Humano. Valencia: Cristóbal Serrano. 
EtXebarria, I. (1989). Contradicciones en los momentos de cambio de valores morales. En Dir. Erxeberría, Perspectivas acerca del cambio moral, posibles intervenciones educativas. San Sebastian: Servicio Editorial de la Universidad del País Vasco.

Freeman, S. J., y Giebinik, J. W. (1979). Moral Judgement as a Function of Age, Sex, and Stimulus. Journal of Psychology, 102, 43-47.

GILIGaN, C. (1982). In a Different Voice. Cambridge: Harvard University Press.

Habermas, J. (1985). Conciencia moral y acción comunicativa. Barcelona: Peninsula.

Hoffman, M. (1977). Moral Internation: Current Theory and Research. En Berkowitz (ed), Advances in Experimental Social Psychology, V.10. Nueva York: Academic Press.

JORDAN, J. A., y SANTOLARIA, F. F. (1987). La educación moral, boy, cuestiones y perspectivas. Barcelona: PPU.

KOHLBERG, L. (1981). The philosopby of moral development. San Francisco: Harper and Row.

KOHLBERG, L. (1992). Psicología del desarrollo moral. Bilbao: Desclée de Brouwer.

Magowan, S. A., y Lee, T. (1970). Some sources of Error in the Use of the Pryective Method for Assessment of Moral Judgement. British Joumal of Psychology, 61, 535-543.

Martínez, B. (1991). Evolución de las diferencias entre sexos en variables psicopedagógicas. Tesis doctoral. Universidad del Pais Vasco.

MartíneZ, M., y Puig, J. M. (1991). La educación moral. Perspectivas de futuro y técnicas de trabajo. Barcelona: Graó.

MEDR ANO, C. (1986). Adquisición de los estadios de razonamiento moral y diferencias en su uso, ante situaciones bipotéticas y situaciones reales en sujetos de 9 a 22 años. Bilbao: Servicio Editorial de la Universidad del País Vasco.

MEDRano, C. (1990). Un modelo cognitivo para la explicación del desarrollo socio-moral: aportaciones y límites. Revista Vasca de Psicología. V.III, 57-61.

Medrano, C.; de la Caba, M. A., y Parra, P. (1991). Actas del Primer Congreso Internacional de Psicología y Educación, Noviembre, Madrid.

Medrano, C., y EleXPURU, I. (1994). Las ideas previas en torno al concepto de valor y su relevancia para el trabajo institucional. En Clemente y otros. Intervención educativa y desarrollo bumano. Valencia: Cristóbal Serrano.

Medrano, C., y DE LA CABA, M. A. (19941). A model of Intervention for improving Moral reasoning: An Experiment in the Basque Country. Moral Education, V.23, 4, 427-437.

MolTo, J., y otros (1990). La variable sexo y su efecto en el desarrollo estructural y de contenido del razonamiento moral. Revista de Pensament $i$ Anàlisi, 15 (1-2).

NaISBITT, J. (1983). Macrotendencias: diez nuevas orientaciones que están transformando nuestras vidas. México: Mitre.

PÉrez-Delgado, E., y Garcia-Ros, R. (1991). La psicología del desarrollo moral. Madrid: Siglo XXI.

PETERS, R.S. (1984). Desarrollo moral y educación moral. México: ECE

PIAGET, J. (1932)(1977). El criterio moral en el niño. Barcelona: Fontanella.

PuIG, J. M., y MARTINEZ, M. (1989). Educación moral y Demacracia. Barcelona: Laertes.

RAwLS, J. (1979). La teoría de la justicia. Madrid: Fondo de Cultura Econónica.

ReST, J. (1979). Development in judging moral issues. Minneapolis: University Press.

WALKER, L. (1984). Sex Differences in the Development of Moral Reasoning: A Critical Review. Child Development, 55, 677-691. 


\section{Las diferencias en el desarrollo moral en función del sexo: un estudio realizado en el País Vasco Concepción Medrano Samaniego CL\&E, 1995, 28, pp. 37-48}

Resumen: En este trabajo, se ha tratado de conocer las diferencias de sexo, en cuanto al razonamiento moral. Después de presentar los modelos e investigaciones que han estudiado este tema, se presentan los datos de 250 sujetos (125 varones y 125 hembras) agrupados en cinco grupos; desde la edad de 8 años hasta los 25 .

El método aplicado se ha basado en la entrevista clínica de Kohlberg. Después de pasar un dilema hipotético, se ha solicitado al sujeto que nos relate un dilema real. En el análisis de resultados, no se encuentran diferencias entre sexos, en cuanto al razonamiento estructural. Sin embargo, si aparecen diferencias en cuanto a los contenidos y valores que exponen. Estos datos presentan un gran interés para poder trabajar los contenidos transversales, referidos a la educación moral.

Datos sobre la autora: Lleva trabajando en el área de valores morales, desde el enfoque cognitivo-evolutivo, desde 1986. Ha colaborado con M.J. Díaz-Aguado en varias investigaciones en este campo; como fruto de esta colaboración se ha publicado por el ICE de la Universidad de Deusto (Bilbao) el texto: Educación y desarrollo moral. Una aproximación constructivista para trabajar los contenidos transversales (1994).

Dirección: Universidad del País Vasco. Avenida de Tolosa, 70. 20009 San Sebastián.

C PERMISOS PARA CITAR O REPRODUCIR EN OTRAS FUENTES: Se pueden citar libremente hasta 500 palabras. Para reproducir una porción de texto mayor, figuras o ilustraciones, se deberá pedir permiso por escrito a la revista, especificando el uso al que se destina el texto. En todos los casos, se deberá citar el copyright de $C L \& E$. En el caso de artículos o textos que hayan sido a su vez reproducidos en $C L \& E$ los interesados deberán dirigirse tanto a los detentadores del copyright original como a $C L \& E$, en el caso de que se quiera hacer uso de la traducción. FOTOCOPIAS: Para todo lo relacionado con el uso mediante fotocopia del material de esta revista, deberán dirigirse a: CEDRO, C/ José Marañón, 10, 3. ${ }^{\circ}$ Izda. Tel. 5941575 . Fax 4453567 